\title{
APPLICATIONS OF THE INTEGRAL OF AN INVARIANT OF THE HESSIAN
}

\author{
BY ROBERT C. REILLY
}

Communicated by S. S. Chern, March 5, 1976

In this paper we announce several results whose proofs will appear elsewhere.

Throughout this paper $M$ will be an $n$-dimensional smooth, connected, compact and oriented Riemannian manifold with boundary $N$.

Definition. If $f$ is a smooth function on $M$ then the $r$ th invariant, $S_{r}(f)$, of the Hessian operator of $f$, where $r=0,1, \ldots, n$, is the $r$ th elementary symmetric function of the eigenvalues of this operator.

THEOREM. 1. If $M, N$ and $f$ are as above then

$$
\begin{aligned}
\int_{M} 2 S_{2}(f) \Omega= & \int_{N}\left\{\left(\Delta z-u K_{1}\right) u-\langle\nabla z, \nabla u\rangle-\operatorname{II}(\nabla z, \nabla u)\right\} \Psi \\
& +\int_{M} \operatorname{Ric}(\operatorname{grad} f, \operatorname{grad} f) \Omega .
\end{aligned}
$$

In this formula $z=\left.f\right|_{N}, u$ is the exterior derivative of $f$ along $N, K_{1}$ is $n-1$ times the mean curvature and II is the second fundamental form (of $N$ in $M$ ), while $\Delta$ is the Laplace operator, $\nabla$ is the gradient and $\Psi$ is the volume form on $N$; similarly, grad is the gradient, Ric is the Ricci tensor and $\Omega$ is the volume form on $M$.

REMARK. We have a similar formula involving $S_{r}(f)$ for $r \geqslant 3$.

THEOREM 2. Let $M, N$ be as above and assume that (a) for some constant $c^{2}>0$ all the Ricci curvatures on $M$ are bounded below by $(n-1) c^{2}$ and (b) $K_{1} \leqslant 0$. Then the first eigenvalue $\lambda_{1}$ for the Laplace operator on $M$ (for the fixed membrane problem) satisfies the inequality $\lambda_{1} \geqslant n c^{2}$. Moreover, equality occurs if and only if $M$ is isometric to a full hemisphere of the standard sphere of radius $1 / c$.

REMARK. This generalizes to the situation of manifolds with boundary the well-known theorems of Lichnerowicz [3] and Obata [4]. Our proof uses Theorem 1 and an analog of Obata's Theorem A.

COROLlaRY. Let $N$ be a compact minimally imbedded hypersurface in the $n$-sphere of radius $1 / c$ and let $M$ be one of the two domains into which $N$ cuts the sphere. If $\lambda_{1}$ is the first eigenvalue of the Laplace operator on $M$, then $\lambda_{1} \geqslant$ $n c^{2}$, with equality if and only if $N$ is imbedded as an equator.

$A M S(M O S)$ subject classifications (1970). Primary 53C20, 53C40.

Copyright $\odot 1976$, American Mathematical Society 
Theorem 1 also provides a simple proof of the following well-known theorem of Aleksandrov [1].

THEOREM 3. If $N$ is a compact hypersurface in $\mathbf{R}^{n}$, with constant mean curvature, which bounds a compact domain $M$, then $N$ is a hypersphere.

In our proof of Theorem 3 we apply Theorem 1 to a solution of the Poisson equation $\Delta f=1$, with boundary condition $f=0$ on $N$.

Our final application of Theorem 1 extends results of Flanders [2].

THEOREM 4. Let $W$ be an n-dimensional Riemannian manifold of positivesemidefinite Ricci curvature. For a point $p$ in $W$ and real $t>0$ let $B(p, t)$ and $S(p, t)$ denote (respectively) the closed ball and the sphere of radius $t$ about $p$. Suppose that $T>0$ is small enough that for all $t \in(0, T], B(p, t)$ is a normal neighborhood of $p$ and $S(p, t)$ has negative semidefinite second fundamental form (relative to the exterior normal to $B(p, t)$ ). Denote the volume of $B(p, t)$ by $V(t)$. If $f$ is a smooth function which satisfies on $B(p, t)$ an inequality $S_{2}(f)$ $\leqslant-\alpha\left(1+|\operatorname{grad} f|^{2}\right)^{1+2 \epsilon}$ for certain positive constants $\alpha$ and $\epsilon$, then for each $t_{1} \in(0, T]$ we have

$$
1 \leqslant \epsilon\left(\frac{2 \alpha}{1+\epsilon}\right)^{1 / 2} \int_{t_{1}}^{T}\left(\frac{V\left(t_{1}\right)}{V(t)}\right)^{\epsilon} d t .
$$

Our proof uses Theorem 1 (with $M=B(p, T)$ ) together with a generalization of formula 3.1 of [2].

REMARK. Flanders proves (2) in the special case $W=\mathbf{R}^{n}$ and uses it to estimate (in terms of $\alpha, \epsilon$ and $n$ ) an upper bound on $T$. We are able to obtain similar bounds in certain other cases, for example:

COROLlary. Suppose that $W$ is a two-dimensional sphere of radius $R$. If there is a function $f$ which satisfies the hypotheses of Theorem 4 with $\epsilon=1$, then $T \leqslant 4 R \operatorname{arc} \sin \left(1 /\left(1+R^{2} \alpha\right)^{1 / 2}\right)$.

REMARK. We arrive at the estimate obtained by Flanders in the corresponding case $W=R^{2}, \epsilon=1$, by letting $R$ tend towards infinity.

\section{REFERENCES}

1. A. D. Aleksandrov, Uniqueness theorems for surfaces in the large. V, Vestnik Leningrad Univ. (13) (1958), no. 19, 5-8; English transl. Amer. Math. Soc. Transl. (2) 21 (1962), 412-416. MR 21 \#909; 27 \#698e.

2. H. Flanders, Non-parametric hypersurfaces with bounded curvatures, J. Differential Geometry 2 (1968), 265-277. MR 39 \#2097.

3. A. Lichnerowicz, Géométrie des groupes de transformations, Dunod, Paris, 1957. MR 23 \#A1329.

4. M. Obata, Certain conditions for a Riemannian manifold to be isometric with a sphere, J. Math. Soc. Japan 14 (1962), 333-340. MR 25 \#5479.

DEPARTMENT OF MATHEMATICS, UNIVERSITY OF CALIFORNIA, IRVINE, CALIFORNIA 92717 